\title{
Non-native Chelonians in the National Zoological Collections of Zoological Survey of India
}

\author{
Shantanu Kundu' ${ }^{1}$, Vikas Kumar ${ }^{1 *}$, B. H. C. K. Murthy² and Kailash Chandra ${ }^{1}$ \\ 'Zoological Survey of India, M - Bolck, New Alipore, Kolkata - 700053, \\ West Bengal, India; vikaszsi77@gmail.com \\ ${ }^{2}$ Western Ghat Regional Centre (WGRC), Zoological Survey of India, Kozhikode - 673006, Kerala, India
}

\begin{abstract}
The native species is the biological assets of a nation, however the non-native species is the threat for indigenous taxa. Thus, before describing any native or non-native species, it is required to thoroughly check the collateral information. As of now, many non-native species from different faunal group were invaded into the native eco-system and reported from India. In this study, we represent the check-list of non-native turtles and tortoises stored in National Zoological Collections of Zoological Survey of India to assure their taxonomic rank and distribution pattern. The list is enriched with the registration numbers, most recent species name, collection localities, conservation status, and other taxonomic information. This comparative data of 35 non-native turtles not only useful in taxonomic research, but also helpful to recognize the invasive species from India and quarantine regulation.
\end{abstract}

Keywords: Archival specimens, Conservation, Ecosystem, Exotic species, Taxonomy

\section{Introduction}

National Zoological Collections (NZC) of the Zoological Survey of India (ZSI) is the largest repositories of zoological specimens in India, holding a huge number of chelonians. Extensive surveys and explorations of faunal diversity within India and adjacent countries since the early 1800s have let ZSI to recognize as the most significant organization in systematic zoology in India. A total of 1173 turtle specimens were preserved in ZSI, in wet as well as in dry condition (Lehn et al., 2007). Many paleontological and zoological materials of turtles were collected from all over British India and often included from Middle East, Africa, North America, Central Asia, the Malayan Peninsula and Archipelago, and Eastern China. The collections and preservation of native as well as non-native turtle species was made by the naturalists during the scientific expeditions. Many non-native taxa were also received from museums such as Berlin, London, Karachi and Port Louis via exchanges or as donations (Murthy and Das, 2009). Such resources may be of dynamic importance being Holotype or other types, extant threatened species or extinct one. Briefly, the ZSI has provided an over-the-top contribution of chelonian research around the globe.

However, the contents of the non-native chelonian collections of the ZSI had remained unknown to the outside world due to non-availability of a catalogue. Although Das et al., 1998, documented reptile types in the collection of the ZSI, followed by a checklist on the voucher of collections in the ZSI by Murthy and Das in 2009, which however lack of comprehensive information of non-native chelonian species. In taxonomic research, the use of integrative approaches in the delineation of species and/or subspecies is not uncommon. Thus, over time, species concept, criteria, and the morphological characters used to differentiate species may alter (Sharon et al., 2006). The archival data provide researchers with the option to revisit morphological characters or advancing technologies to re-examine the previous descriptions or conclusions (Stuckas et al., 2013). The recent study suggested that the existence of three non-native turtle species, Cyclemys fusca, Amyda ornata and Chitra chitra in northeastern region of India evidenced by genetic information (Kundu et al., 2016). Further, the illegal trade of 'red-eared slider turtle'Trachemys scripta elegans,

\section{* Author for correspondence}


native to western nations, is clearly established by the two arrested passengers on $19^{\text {th }}$ July 2013 by Indian Customs Officials at the Netaji Subhas Chandra Bose International Airport in Kolkata. The biggest consignment of 10,043 non-native turtles were identified by the ZSI scientists and suspected of smuggling from China to Kolkata via Singapore. Furthermore, on 21 August 2015, The Times of India reported the same species from a water body within the Kolkata city premise in India. Thus, the information, especially the collection localities of a non-native chelonian further furnished a scope of expanding the range distribution in India as well as other geographical regions beyond the existing thoughts. The data of archival taxa further impelled to review their real existence or accurate distribution in wild as well as reassessment of any species by IUCN/SSC Tortoise and Freshwater Turtle Specialist Group (TFTSG) or other international organization, involved in the field of nature conservation and sustainable use of natural resources. Besides, the data of a non-native species also help to understand their native ranges to protect native species from havoc in same environment.

This research notes present a list of all non-native chelonian collections in the Zoological Survey of India, Kolkata. The table represents the registration numbers of the non-native chelonians, species name (nomenclature follows Fritz and Havaš, 2007, IUCN Red data List, http:// www.iucnredlist.org/ and The Reptile Database, http:// reptile-database.reptarium.cz/) and authors, collection localities and IUCN status (IUCN, 2017). The study encompasses 35 non-native chelonian species of seven families from the archival collections of ZSI. The related data were collected from the 'Species Card', 'Species Register' and from the tag of the available specimens and compared with the previous literatures. The figure represents the representative of non-native chelonian species preserved in National Zoological Collections of ZSI. The morphometric measurement of carapace and plastron were taken by Vernier slide calipers and photographs of Chelydra serpentina were taken by Nikon D3300 (AF-S Nikkor 8-55 mm lens). Further, the detailed morphology and additional molecular data of the studied specimens would substantiate to know the actual systematics to resolve many taxonomic quests.

\section{Remarks}

The collateral data of non-native Chelonians in Zoological Survey of India reflects the holding of archival collection in National Zoological Collections. The collection localities of a few species mentioned in Species Card or Species Register further obligate to revisit the morphological characters of preserved species. The species collected from Calcutta (=Kolkata) markets as well as from Zoological Garden, Calcutta, are might be donated sold by someone in local markets. Thus, the

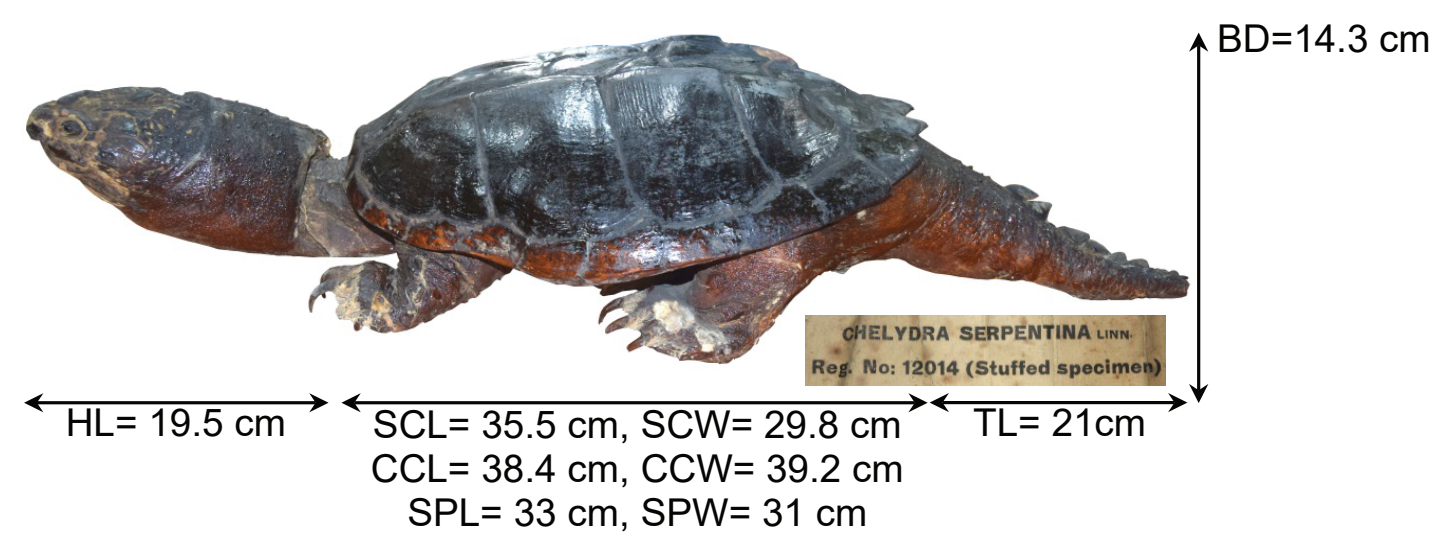

Figure 1. Representative of exotic Chelonians (the common snapping turtle, Chelydra serpentina) preserved in Zoological Survey of India, Kolkata, species tag and morphometric measurements. HL = Head Length, SCL = Straight Carapace Length, SCW = Straight Carapace Width, CCL= Curved Carapace Length, CCW- Curved Carapace Width, SPL = Straight Plastron Length, SPW= Straight Plastron Width, BD = Body Depth. 
Table 1. List of exotic chelonians in NZC of Zoological Survey of India, Kolkata. The registration numbers and collection localities were verified from the species card and ZSI registered

\begin{tabular}{|c|c|c|c|c|}
\hline Sl. No. & Species Name and Authority & $\begin{array}{l}\text { IUCN status } \\
2016-3\end{array}$ & $\begin{array}{l}\text { Registration Numbers in Reptil- } \\
\text { ia section }\end{array}$ & Collection localities \\
\hline \multicolumn{5}{|c|}{ Family: Testudinidae } \\
\hline 1 & $\begin{array}{l}\text { Aldabrachelys gigantea (Sch- } \\
\text { weigger, 1812) }\end{array}$ & $\begin{array}{l}\text { Not assessed, } \\
\text { Extinct species }\end{array}$ & 14921 & Mauritius \\
\hline 2 & $\begin{array}{l}\text { Chersina angulata } \\
\text { (Schweigger, 1812) }\end{array}$ & Not assessed & 808 (8a A.S.B) & South Africa \\
\hline 3 & $\begin{array}{l}\text { Manouria impressa } \\
\text { (Günther, 1882) }\end{array}$ & Vulnerable & $663(1140)$ & Pegu, Burma \\
\hline 4 & $\begin{array}{l}\text { Testudo marginata } \\
\text { (Schoepff, 1793) }\end{array}$ & Least Concern & 16724 & Zoological Garden, Calcutta \\
\hline 5 & $\begin{array}{l}\text { Astrochelys radiata } \\
\text { (Shaw, 1802) }\end{array}$ & $\begin{array}{l}\text { Critically Endan- } \\
\text { gered }\end{array}$ & 804 (2a A.S.B); 15487 & South Africa; Madagascar \\
\hline 6 & $\begin{array}{l}\text { Testudo hermanni } \\
\text { (Gmelin, 1789) }\end{array}$ & Near Threatened & $\begin{array}{l}507(42) ; 508(44) ; 509 ; 510(51) ; \\
515(41) ; 516 ; 517(49) ; 518(46)\end{array}$ & Not Known \\
\hline 7 & $\begin{array}{l}\text { Testudo graeca } \\
\text { (Linnaeus, 1758) }\end{array}$ & Vulnerable & $\begin{array}{l}300 ; 301 ; 519(47) ; \\
11346 ; 11347 ; 11348\end{array}$ & $\begin{array}{l}\text { Karman, South East Arabia; Aun- } \\
\text { sb-Tin, near Lake Galille }\end{array}$ \\
\hline 8 & $\begin{array}{l}\text { Testudo horsfieldi } \\
\text { (Gray, 1844) }\end{array}$ & Not assessed & $\begin{array}{l}\text { 3856; 5591; 5592; 5593; 5594; } \\
\text { 5595; } \\
\text { 5596; 5597; 5598; 5599; 5600; } \\
\text { 15538; 15541; 15542; 15543; } \\
\text { 16478; 16479; 16480; 15551; } \\
\text { 15552; } 793 \text { (7a A.S.B); } 11420\end{array}$ & $\begin{array}{l}\text { Safed-i-Rak, Kabul; } \\
\text { Kelat, Baluchistan; } \\
\text { Quetta; } \\
\text { Khanai, Quetta; Afghanistan }\end{array}$ \\
\hline 9 & $\begin{array}{l}\text { Geochelone platynota } \\
\text { (Blyth, 1863) }\end{array}$ & $\begin{array}{l}\text { Critically Endan- } \\
\text { gered }\end{array}$ & $\begin{array}{l}988(1137) ; 2653(181) ; 17049 \\
732 ; 787 \text { (5a A.S.B.); } 788 \text { (5b } \\
\text { A.S.B); } 789 \text { (5c A.S.B) }\end{array}$ & $\begin{array}{l}\text { Akyal, Burma; } \\
\text { North Pegu, Burma, Irrawaddy } \\
\text { River near Yenangyat, Pukokuku } \\
\text { Dist. Burma; Upper Burma }\end{array}$ \\
\hline 10 & $\begin{array}{l}\text { Indotestudo forstenii } \\
\text { (Schlegel and Müller, 1845) }\end{array}$ & Endangered & $17697 ; 17017 ; 18045 ; 24520$ & $\begin{array}{l}\text { Cochin states forest, Kerala; } \\
\text { Punalur, Travancore, Kerala }\end{array}$ \\
\hline \multicolumn{5}{|c|}{ Family: Geoemydidae } \\
\hline 11 & $\begin{array}{l}\text { Mauremys japonica } \\
\text { (Temminck and Schlegel, } \\
1835 \text { ) }\end{array}$ & $\begin{array}{l}\text { Lower Risk/Near } \\
\text { Threatened }\end{array}$ & 1418 & Japan \\
\hline 12 & Heosemys spinosa (Gray, 1830) & Endangered & 11693 & Serawak \\
\hline 13 & $\begin{array}{l}\text { Heosemys annandalii (Bou- } \\
\text { lenger in Annandale and } \\
\text { Robinson, 1903) }\end{array}$ & Endangered & 18914 & Western Bangkok, Siam \\
\hline 14 & $\begin{array}{l}\text { Batagur trivittata } \\
\text { (Duméril and Bibron, 1835) }\end{array}$ & Endangered & $744 ; 1443$ & Burma \\
\hline 15 & $\begin{array}{l}\text { Notochelys platynota } \\
\text { (Gray, 1834) }\end{array}$ & Vulnerable & $\begin{array}{l}\text { 1021; } 1021 ; 1323(792) ; 1326 \\
\text { (934); }\end{array}$ & Burma \\
\hline
\end{tabular}




\begin{tabular}{|c|c|c|c|c|}
\hline 16 & $\begin{array}{l}\text { Heosemys depressa (Anderson, } \\
1875 \text { ) }\end{array}$ & $\begin{array}{l}\text { Critically Endan- } \\
\text { gered }\end{array}$ & $\begin{array}{l}490(1008) ; 1176(1309) ; 1319 \\
(2303) ; \\
1322(1656)\end{array}$ & Arakan, Burma \\
\hline 17 & $\begin{array}{l}\text { Mauremys reevesii } \\
\text { (Gray, 1831) }\end{array}$ & Endangered & $\begin{array}{l}\text { 18028; } 18029 ; 18030 ; 18031 ; \\
18032 ; 18033\end{array}$ & $\begin{array}{l}\text { Tong-Dong-Ding, Tai- Hu, Ki- } \\
\text { angsu Province, China; } \\
\text { Tai- Hu, Kiangsu Province, China }\end{array}$ \\
\hline 18 & $\begin{array}{l}\text { Mauremys caspica (Gmelin, } \\
1774 \text { ) }\end{array}$ & Not assessed & $\begin{array}{l}\text { 18892; 18893; 18894; 296;297; } \\
\text { 298;299; 505; 19238; 18894; } \\
22952 ; 23714\end{array}$ & $\begin{array}{l}\text { Nasiriyeh Euphratis River, Meso- } \\
\text { potomia; } \\
\text { North Persia; } \\
\text { Caspian Sea; Shatta- el- Arab, } \\
\text { Iraq; Central Iraq }\end{array}$ \\
\hline 19 & Cyclemys dentata (Gray, 1831) & $\begin{array}{l}\text { Lower Risk/near } \\
\text { threatened }\end{array}$ & $\begin{array}{l}760(1438) ; 775(408) ; 18568 ; \\
1332(901) ; 18593 ; 18594 ; 19235 ; \\
20023 ; 20449 ; 335 ; 339 ; 340 ; \\
341 ; 1328 ; 1329 ; 1330 ; 1333 ; \\
18482 ; 13 ; 410 ; 12603 ; 820 ; \\
821 ; 994(753) ; 1356(544) ; 1320 \\
(1484) ; 1325(1300) ; 1327(1926)\end{array}$ & $\begin{array}{l}\text { Burma; Tura, Garo Hills, Assam; } \\
\text { He-Hah, South Shan states; } \\
\text { Fort Stedman Inte Lake, South } \\
\text { Shan states; Sattau Chaung } \\
\text { stream Myitkyina, Dist. Upper } \\
\text { Burma; } \\
\text { Narainpur Tea Estate, Cachar, } \\
\text { Assam; Calcutta, India market; } \\
\text { Akyab, Burma; Sibsagar, Assam; } \\
\text { Joranti River, West Dooars; Ara- } \\
\text { kan Hills, Burma; } \\
\text { Tenasserium, Burma }\end{array}$ \\
\hline 20 & Heosemys grandis (Gray, 1860) & Vulnerable & $\begin{array}{l}918(354) ; 920(361) ; 921(375) ; \\
922(388) ; 13008(2006) ; 1783 \\
(2469) ; 2052 ; 923(406) ; 1309 ; \\
1311(1626) ; 1307(2179) ; 20474 ; \\
916(347) ; \\
1310(2065) ; 1315(2910) ; 1316 \\
(2495) ; \\
1487(2318) ; 1490(2529) ; 699 \\
(410) ; 917(351) ; 926(436) ; 927 \\
(439) ; 815 ; 2052 ; \\
710(388) ; 729 \\
\end{array}$ & $\begin{array}{l}\text { Burma; Enoshima, Japan; Tenas- } \\
\text { serim, Burma }\end{array}$ \\
\hline 21 & $\begin{array}{l}\text { Siebenrockiella crassicollis } \\
\text { (Gray, 1830) }\end{array}$ & Vulnerable & $\begin{array}{l}648(301) ; 834(16 a \text { A.S.B }) ; 835 \\
(16 b \text { A.S.B }) ; 1378 ; 18039 ; 18040 ; \\
15 ; 3862 ; 18023 ; 18024 ; 567 \\
(933) ; 639(322) ; 764(1073)\end{array}$ & $\begin{array}{l}\text { Burma; Lanpung, Patalung, Siam; } \\
\text { Penang; } \\
\text { Singgora, Siam; } \\
\text { Lungpung, Patalung, Siam }\end{array}$ \\
\hline 22 & $\begin{array}{l}\text { Morenia ocellata (Duméril } \\
\text { and Bibron, 1835) }\end{array}$ & Vulnerable & $\begin{array}{l}178 ; 179 ; 180 ; 181 ; 182 ; 183 ; \\
185 ; 186 ; 187 ; 191 ; 199 ; 203 \\
(364) ; 206(59) ; 207(377) ; 208 \\
(386) ; 209(298) ; 211 ; 1601 \\
(321) ; 946(223) ; 1460(295) ; \\
1464(418) ; 935 ; 939(129) ; 1019 \\
(408) ; 1468(313) ; 1469(283) ; \\
1470(204) ; 1483(146) ; 1473 ; \\
2042 ; 859 ; 861 ; 860 ; 862 ; 863 ; \\
864 ; 940(136) ; 941(155) ; 942 \\
(201) ; 947(232) ; 948(233) ; 950 \\
(281) ; 951(284) ; 952\end{array}$ & $\begin{array}{l}\text { Akyab; Burma; } \\
\text { Pegu }\end{array}$ \\
\hline
\end{tabular}




\begin{tabular}{|c|c|c|c|c|}
\hline 23 & $\begin{array}{l}\text { Malayemys subtrijuga } \\
\text { (Schlegel and Müller, 1845) }\end{array}$ & Vulnerable & $\begin{array}{l}\text { 18025; } 18027 ; 18041 ; 824(13 a \\
\text { A.S.B); } 825 \text { (13b A.S.B); } 826(13 c \\
\text { A.S.B); } 18041\end{array}$ & $\begin{array}{l}\text { Lampam, Patalung, Siam; } \\
\text { Singgora, Siam; } \\
\text { Java }\end{array}$ \\
\hline 24 & $\begin{array}{l}\text { Mauremys rivulata (Valenci- } \\
\text { ennes, 1833) }\end{array}$ & Not assessed & $\begin{array}{l}11349 ; 11350 ; 11351 ; 11352 \\
11353 ; 17055 ; 17056\end{array}$ & $\begin{array}{l}\text { Sea of Galifee; } \\
\text { West of Es-semakh, lake, Pales- } \\
\text { tine; } \\
\text { Palestine }\end{array}$ \\
\hline \multicolumn{5}{|c|}{ Family: Trionychidae } \\
\hline 25 & $\begin{array}{l}\text { Pelochelys bibroni } \\
\text { (Owen, 1853) }\end{array}$ & Vulnerable & 20478 & Calcutta (market) \\
\hline 26 & $\begin{array}{l}\text { Pelodiscus sinensis (Wieg- } \\
\text { mann, 1835) }\end{array}$ & Vulnerable & $\begin{array}{l}279 ; 4693 ; 4694 ; 4695 ; 18034 ; \\
18035 ; 18036 ;\end{array}$ & $\begin{array}{l}\text { Shanghai, China; } \\
\text { Hiknoe Lake, Biwa, Japan; } \\
\text { Taittu, Kiangsu Province, China }\end{array}$ \\
\hline 27 & $\begin{array}{l}\text { Dogania subplana (Geoffroy } \\
\text { Saint-Hilaire, 1809) }\end{array}$ & $\begin{array}{l}\text { Lower Risk/least } \\
\text { concern }\end{array}$ & $661(1010) ; 11589 ; 13468 ; 13469$ & $\begin{array}{l}\text { Sinkipls, East coast of Sumatra; } \\
\text { Kingsls. Mergui, Archipelago }\end{array}$ \\
\hline 28 & $\begin{array}{l}\text { Nilssonia formosa } \\
\text { (Gray, 1869) }\end{array}$ & Endangered & $\begin{array}{l}1051 ; 1785 ; 1786 ; \\
688(943) ; 634 ; 1785 ; 1786 ; \\
1787 ; 1837 ; 766 ; 277 ; 278 ; 687 \\
(942)\end{array}$ & $\begin{array}{l}\text { Burma; Moulmein; } \\
\text { Irrawaddy River; } \\
\text { Mandaley }\end{array}$ \\
\hline \multicolumn{5}{|c|}{ Family: Emydidae } \\
\hline 29 & $\begin{array}{l}\text { Chrysemys picta (Schneider, } \\
1783 \text { ) }\end{array}$ & Least Concern & 418 & North America \\
\hline 30 & $\begin{array}{l}\text { Trachemys scripta (Schoepff, } \\
\text { 1792) }\end{array}$ & Least Concern & $444 ; 3860$ & North America \\
\hline 31 & $\begin{array}{l}\text { Clemmys guttata } \\
\text { (Schneider, 1792) }\end{array}$ & Endangered & $395 ; 397$ & North America \\
\hline 32 & $\begin{array}{l}\text { Emys orbicularis (Linnaeus, } \\
1758 \text { ) }\end{array}$ & $\begin{array}{l}\text { Lower Risk/near } \\
\text { threatened }\end{array}$ & $302 ; 485 ; 978$ & $\begin{array}{l}\text { Enzelion on Caspian Sea; } \\
\text { Berlin Museum; }\end{array}$ \\
\hline \multicolumn{5}{|c|}{ Family: Kinosternidae } \\
\hline 33 & $\begin{array}{l}\text { Kinosternon subrubrum } \\
\text { (Lacépède, 1788) }\end{array}$ & Least Concern & $391(1559)$ & North America \\
\hline \multicolumn{5}{|c|}{ Family: Chelydridae } \\
\hline 34 & $\begin{array}{l}\text { Chelydra serpentina (Linnae- } \\
\text { us, 1758) }\end{array}$ & Least Concern & $12014 ; 426 ; 12288 ; 12293$ & $\begin{array}{l}\text { Karachi; } \\
\text { North America }\end{array}$ \\
\hline \multicolumn{5}{|c|}{ Family: Platysternidae } \\
\hline 35 & $\begin{array}{l}\text { Platysternon megacephalum } \\
\text { (Gray, 1831) }\end{array}$ & Endangered & $873 ; 205 ; 16040 ; 16720$ & $\begin{array}{l}\text { Martaban, Burma; } \\
\text { Tonghu, Burma; } \\
\text { Dawna Hills, Lower Burma; } \\
\text { Sukli East side of Dawana Hills, } \\
\text { Lower Burma }\end{array}$ \\
\hline
\end{tabular}

exact collection localities of that species are unknown. Further, in two genera, viz., Cyclemys and Indotestudo, the distribution information of their congeners is very poor. In recent reviews, the diversity of Cyclemys congeners were described in detail: C. atripons are distributed in South-eastern Thailand, including the Ko Chang and Ko Kut islands, South-western Cambodia; $C$. dentata are distributed in Southern Malay Peninsula, Sumatra, Java, Borneo and nearby small islands, Palawan Islands and Sulu Archipelago, Philippines, introduced in Leyte and some other islands of the Philippines; C. pulchristriata are distributed in Central and Southern Vietnam; Easternmost Cambodia; C. oldhamii are distributed in Central and Southern Myanmar (Burma), Central and 
Northern Thailand, Laos, Northern Cambodia, Northern and Central Vietnam, perhaps neighboring Southern China; C. enigmatica from Malaysia to Sumatra, Java and up to Borneo, Indonesia; C. fusca from Myanmar; and C. gemeli from Northeast India (Fritz et al., 2008; Praschag et al., 2009; Kundu et al., 2016). Furthermore, the congeners of Indotestudo reportedly inhabit distant geographical locations; I. forstenii is the oldest named species and is believed to be restricted in Sulawesi, Indonesia; I. elongata is distributed through east and northeast India, Myanmar, LAOs, Thailand, Cambodia to Vietnam; and I. travancorica is restricted in South India (Kundu et al., 2012). Thus, the collection localities of C. dentata from Tura, Garo Hills as well as from Sibsagar in Assam states of Northeast India and the collection localities of $I$. forstenii in Cochin states forest, Kerala listed here warrant reexamination of the species identification through indepth morphological studies.

\section{Acknowledgements}

We are grateful to the Director, Zoological Survey of India for his encouragement and moral support and providing necessary facilities. The study is financially supported by the SERB, Department of Science \& Technology, Delhi through National Post-Doctoral fellowship (F. No. PDF/2015/000302) to the first author and Core funding of ZSI to the corresponding author. We thank all the staff in Reptilia section in Spirit Building, ZSI for providing the material for study.

\section{References}

Das I., Dattagupta B. and Gayen, N.C. 1998. History and catalogue of reptile types in the collection of the Zoological Survey of India. Journal of South Asian Natural History, 3: 121-172.

Fritz, U. and Havaš, P. 2007. Checklist of testudines of the world. Vertebrate Zoology, 57: 149-368.

Fritz, U., Guicking, D., Auer, M., Sommer, R.S., Wink, M. and Hundsdörfer, A.K. 2008. Diversity of the Southeast Asian leaf turtle genus Cyclemys: how many leaves on its tree of life? Zoologica Scripta. 37: 367-390.

IUCN. 2017. The IUCN Red List of Threatened Species (Version 2017-2). Available from: http:// www.iucnredlist.org

Kundu, S., Das, K.C. and Ghosh, S.K. 2012. Taxonomic rank of Indian tortoise: Rrevisit with DNA barcoding perspectives. DNA Barcodes, 39-45. DOI: 10.2478/dna-2013-0003.

Kundu, S., Kumar, V., Laskar, B.A., Chandra, K. and Tyagi, K. 2016. Mitochondrial DNA effectively detects non-native Testudines: Invisible wildlife trade in northeast India. Gene Reports, 4: 10-15.

Lehn, C., Das, I., Forstner, M.R.J. and Brown, R.M. 2007. Responsible vouchering in turtle research: an introduction and recommendations. Chelonian Research Monographs, 4: 147-156.

Murthy, B.H.C.K. and Das I. 2009. The turtle collection of Zoological Survey of India, Kolkata, India. Envis Bulletin: Freshwater Turtle and Tortoise of India and Protected Areas, WII, 12: 15-24.

Praschag, P., Hundsdörfer, A.K. and Fritz, U. 2009. Further specimens and phylogenetic position of the recently described leaf turtle species Cyclemys gemeli (Testudines: Geoemydidae). Zootaxa, 29-37.

Sharon, S.Y., Webb, C.O. and Salamin, N. 2006. Exotic taxa less related to native species are more invasive. Proceedings of the National Academy of Sciences of the United States of America, 103: 5841-5845, doi: 10.1073/pnas.0508073103

Stuckas, H., Gemel, R. and Fritz, U. 2013. One extinct turtle species less: Pelusios seychellensis is not extinct, it never existed. PLoS ONE, 8: e57116. doi:10.1371/journal.pone.0057116 